\title{
IR fixed points and conformal window in $S U(3)$ gauge Theories
}

\section{Yu Nakayama*†}

Walter Burke Institute for Theoretical Physics, California Institute of Technology, Pasadena, CA 91125, USA

E-mail: nakayama@theory.caltech.edu

\begin{abstract}
We propose a novel RG method to specify the location of the IR fixed point in lattice gauge theories and apply it to the $S U(3)$ gauge theories with $N_{f}$ fundamental fermions. It is based on the scaling behavior of the propagator through the RG analysis with a finite IR cut-off, which we cannot remove in the conformal field theories in sharp contrast with the confining theories. The method also enables us to estimate the anomalous mass dimension in the continuum limit at the IR fixed point. We perform the program for $N_{f}=16,12,8$ and $N_{f}=7$ and indeed identify the location of the IR fixed points in all cases.
\end{abstract}

The 33rd International Symposium on Lattice Field Theory

14 - 18 July 2015

Kobe International Conference Center, Kobe, Japan*

\footnotetext{
${ }^{*}$ Speaker.

${ }^{\dagger}$ The talk was given on behalf of Prof. Y. Iwasaki.
} 
Scale invariance, or more precisely conformal invariance has become a fundamental concept in understanding the universal aspects of the nature from the Planck scale to the Hubble scale [1] [2]. Obviously, the central question is to locate the IR fixed point within a given class of theories. In this proceeding, we report our proposal presented in [3]. There we have proposed a novel and simple RG method to specify the location of the IR fixed point in lattice gauge theories by studying the scaling behavior of the propagator. We have applied the technique to the $S U(3)$ gauge theories with $N_{f}$ fundamental fermions (within the conformal window [4] [5] [6]), and estimated the anomalous mass dimension. We have completed this program for $N_{f}=16,12,8$ and $N_{f}=7$, and indeed identified the location of the IR fixed points in all cases.

We constructively define gauge theories on Euclidean plane $\mathbf{R}^{4}$ as the continuum limit of lattice gauge theories on the Euclidean lattice of the size $N_{x}=N_{y}=N_{z}=N$ and $N_{t}=r N$ with $r=4$ throughout the article. Our general argument that follows can be applied to any gauge theories with fermions in arbitrary (vector-like) representations, but to be specific, we focus on $S U$ (3) gauge theories with $N_{f}$ fundamental Dirac fermions. For the lattice regularization of the action, we employ the Wilson quark action and the RG improved gauge action[7].

Given the regularized action, the theory is defined by two parameters; the bare coupling constant $g_{0}$ and the bare degenerate quark mass $m_{0}$ at ultraviolet (UV) cutoff. We also use, instead of $g_{0}$ and $m_{0}, \beta=6 / g_{0}^{2}$ and the hopping parameter $K=1 / 2\left(m_{0} a+4\right)$. The hopping parameter is related to the renormalized quark mass $m_{q}$ thorugh the Ward-Takahashi identity.

One of the most important observables we will study is the $t$ dependence of the propagator of the local meson operator in the $H$ channel:

$$
G_{H}(t)=\sum_{x}\left\langle\bar{\psi} \gamma_{H} \psi(x, t) \bar{\psi} \gamma_{H} \psi(0)\right\rangle
$$

where the summation is over all the spatial lattice points. In this paper, we mostly focus on the pseudo-scalar (PS) channel $H=P S$, and the subscript $H$ is suppressed hereafter.
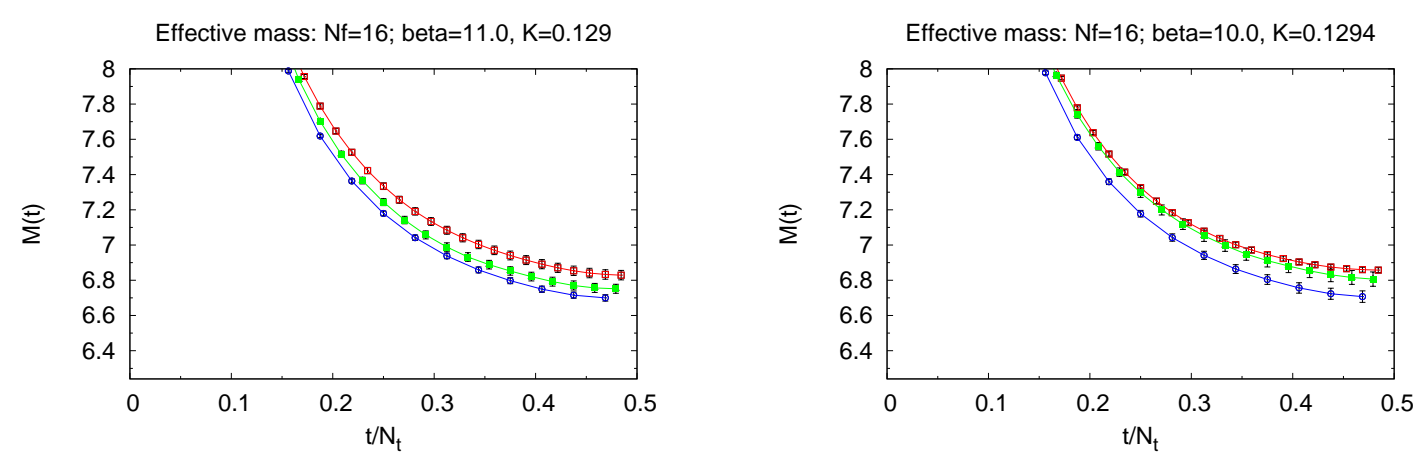

Figure 1: Scaled effective mass plots for $N_{f}=16$ at $\beta=11.0$ and 10.0: three sets of symbols are $N=16$ (red square), $N=12$ (green circle), $N=8$ (blue triangle). 

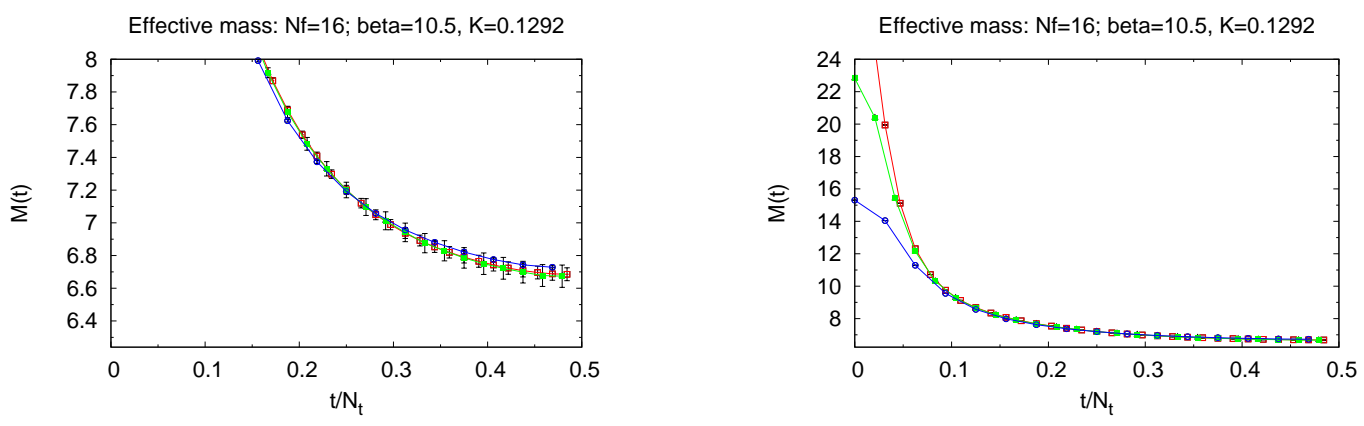

Figure 2: Scaled effective mass plots for $N_{f}=16$ at $\beta=10.5$ : the left panel is an enlarged one of the right panel; three sets of symbols are $N=16$ (red square), $N=12$ (green circle), $N=8$ (blue triangle).

In order to investigate the large $t$ behavior of a propagator, we define the effective mass $m(t)$ through

$$
\frac{\cosh \left(m(t)\left(t-N_{t} / 2\right)\right)}{\cosh \left(m(t)\left(t+1-N_{t} / 2\right)\right)}=\frac{G(t)}{G(t+1)} .
$$

Let us study the RG properties of the propagator in the vicinity of the fixed point. The RG equation for the RG transformation induced by the change of the UV renormalization scale $\mu^{\prime}=$ $\mu / s$, followed by a space-time scale change by a factor $1 / s$ (see e.g. [8]), relates the propagator with different parameters as

$$
G\left(t ; g, m_{q}, N, \mu\right)=\left(\frac{N^{\prime}}{N}\right)^{3-2 \gamma} G\left(t^{\prime} ; g^{\prime}, m_{q}^{\prime}, N^{\prime}, \mu\right) .
$$

Here $N^{\prime}=N / s$ and $t^{\prime}=t / s$. The relation between $g^{\prime}$ and $g$ and $m_{q}^{\prime}$ and $m_{q}$ are determined by the beta function $\mathscr{B}$ and the mass anomalous dimensions $\gamma$.

Let us first discuss the case in which we are at the fixed point, i.e. $g^{\prime}=g=g^{*}$ and $m_{q}^{\prime}=m_{q}=0$ so that $\mathscr{B}=0$ and $\gamma=\gamma^{*}$. In this case, the propagator may have simplified notation as

$$
\tilde{G}(\tau, N)=G(t, N) .
$$

with $\tau=t / N_{t}$. The variable $t$ takes $0,1,2, \cdots, N_{t}-1$ so that $0 \leq \tau \leq 1$. In terms of $\tau$, the RG relation eq. (3) reduces to

$$
\tilde{G}(\tau ; N)=\left(\frac{N^{\prime}}{N}\right)^{3-2 \gamma^{*}} \tilde{G}\left(\tau ; N^{\prime}\right) .
$$

To state our proposal concretely, we define the scaled effective mass $\mathfrak{m}(t ; N)$ as

$$
\mathfrak{m}(t, N)=N \ln \frac{G(t, N)}{G(t+1, N)} .
$$

In the continuum limit $N \rightarrow \infty$ Eq. (6) reduces to the form

$$
\mathfrak{m}(\tau, N)=-\partial_{\tau} \ln \tilde{G}(\tau, N)
$$


The crucial observation, which will be the core of our proposal is that, combining Eqs.(5) and (7), the scaled effective mass does not depend on $N$ as a function of $\tau$ :

$$
\mathfrak{m}(\tau, N)=\mathfrak{m}\left(\tau, N^{\prime}\right)
$$

at the fixed point. Therefore, the agreement of the scaled effective mass as a function of $N$ and $\tau$ are stringent tests of the fixed point.

Our strategy is as follows. With given $N_{f}$ and $\beta$, we tune the quark mass to be zero. Then we numerically compute the meson propagator on the lattice. For each choice of the lattice size $N$, we plot the effective mass defined by Eq. (2) in terms of the scaled time $\tau$. As we explained, generically, the scaled effective mass do not coincide with each other as a function of $\tau$ at a given value of $\beta$ but different values of $N$. However, if we find the fixed point value $\beta$, the plots for different $N$ must coincide with each other.

In this article, we perform numerical simulations on the three lattices with size $8^{3} \times 32,12^{3} \times$ 48 and $16^{3} \times 64$ with the aspect ratio of $r=4$. Let us show in Fig. 1 the scaled effective mass plots in two cases of many such examples: we take $N_{f}=16$ at $\beta=11.0$ and $\beta=10.0$. The asymptotic behaviors of three sets of data points and the lines connecting them on $N=8,12$ and 16 lattices, do not coincide with each other. We may conclude that these values of $\beta$ do not correspond to the fixed point. On the other hand, as we will see in Fig 2, if we take $\beta=10.5$, then the three plots and the lines do coincide within the standard deviation. Based on the RG relations, we claim that this is the value of the gauge coupling constant at the fixed point.

We perform this program for $N_{f}=7,8,12,16$ on lattices with size $8^{3} \times 32,12^{3} \times 48$ and $16^{3} \times 64$. By narrowing down the region where the scaled effective mass $\mathfrak{m}(\tau, N)$ becomes close for different $N$, we identify the point $\beta^{*}$ where they agree with each other within one standard deviation.
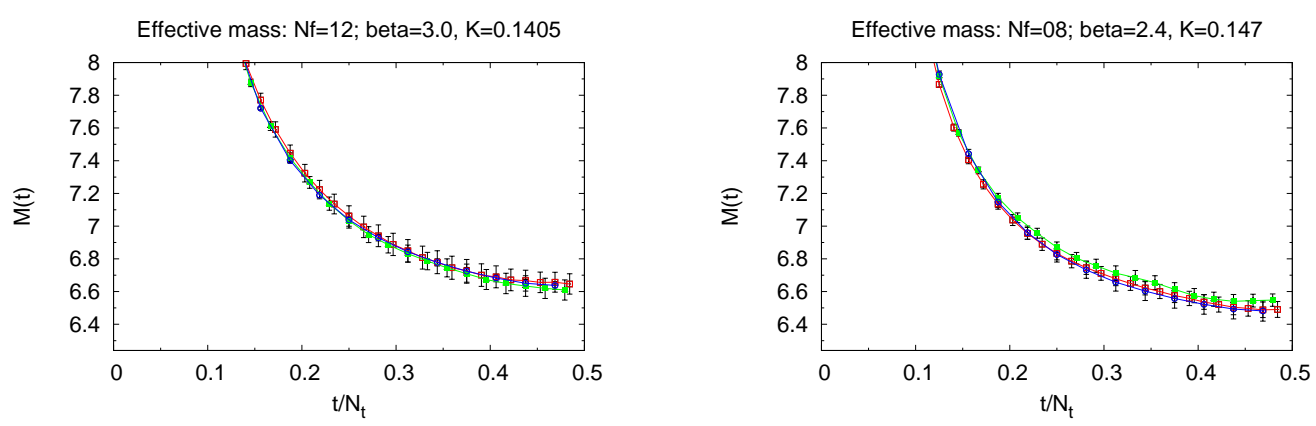

Figure 3: Scaled effective mass plots for $N_{f}=12$ at $\beta=3.0$ and $N_{f}=8$ for $\beta=2.4$; three sets of symbols are $N=16$ (red square), $N=12$ (green circle) and $N=8$ (blue triangle).

The algorithms we employ are the blocked HMC algorithm [9] in the case $N_{f}=2 \mathbb{N}$ and the RHMC algorithm [10] for $N_{f}=1$ in the case $N_{f}=2 \mathbb{N}+1$. The simulation parameters can be found in [3]. 


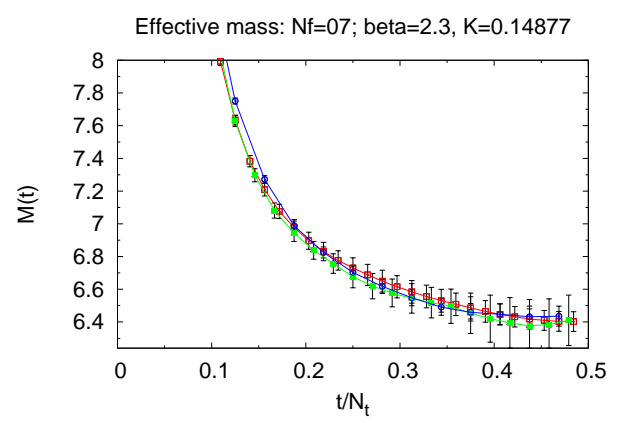

Figure 4: Scaled effective mass plots for $N_{f}=7$ at $\beta=2.3$ : three sets of symbols are $N=16$ (red square), $N=12$ (green circle) and $N=8$ (blue triangle).

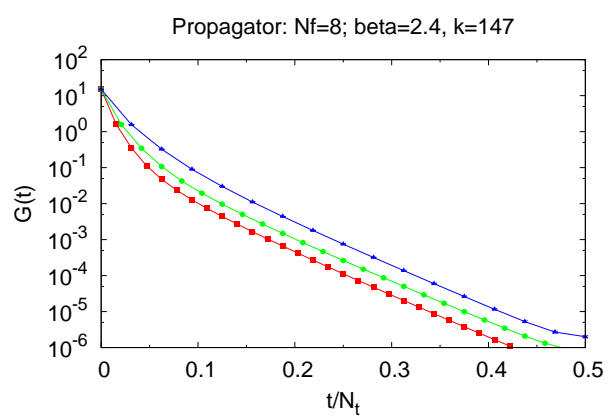

Figure 5: Propagators for $N_{f}=8$ at $\beta=2.4$ : three sets of symbols are $N=16$ (red square), $N=12$ (green circle) and $N=8$ (blue triangle).

Now, let us show the results, starting with the $N_{f}=16$ case. In order to find the fixed point from our proposal,we make several trials including those at $\beta=10.0,10.5,11.0$, and 11.5. We find the three sets of data and the lines connecting them are apparently different from each other at $\beta=11.0$ (Fig. 1; left panel) and they approach closer by decreasing $\beta$ as 11.0,10.5. On the other hand, at $\beta=10.0$ (Fig. 1; right panel) they are apart each other again but they approach closer by increasing $\beta$ as 10.0, 10.5. This suggests that there is an IR fixed point between $\beta=10.0$ and 11.0. We indeed find, as shown in Fig. 2, that the three sets of the scaled effective mass plots are almost degenerate at $\beta=10.5$ and $K=0.1292$. We see that three lines almost overlap for $\tau \geq 0.1$. Only in the small $\tau$ region $(\tau \leq 0.1)$ we see the differences. We interpret the difference for $\tau \leq 0.1$ is due to the fact that $N$ is not large enough to remove the effect of the UV cutoff $\mu=a^{-1}$.

The fact that our method identifies the location of the IR fixed point at a value expected from the perturbation theory [4], together with the fact that three lines almost overlap, strengthens our confidence in the validity of our approach. 
We make similar process for $N_{f}=12,8$ and 7 as the $N_{f}=16$ case. In Figs. 3 and 4 are shown the results. The qualitative feature of our results are the same. If we choose a very particular $\beta$ for each $N_{f}$, the data and three lines almost overlap for $\tau \geq 0.1$, as shown in the Figures. In the small $t$ region $\left(t / N_{t} \leq 0.1\right)$ we find the differences. Since they are similar to the case of $N_{f}=16$, we do not present them here.

Finally we identify the IR fixed points at $\beta^{*}=10.5 \pm 0.5$ for $N_{f}=16 ; 3.0 \pm 0.2$ for $N_{f}=12$; $2.4 \pm 0.1$ for $N_{f}=8$; and $2.3 \pm 0.05$ for $N_{f}=7$.

On the other hand, in the $N_{f}=6$ case, there is a chiral phase transition point at finite $\beta$ when $N$ is finite[11]. If we would perform a program similar to the above (by fixing $\beta$ and increasing the lattice size $N$ ), then at some $N$ the system would end up with the confining phase rather than the chiral symmetric phase (to which the conformal fixed points belong). Thus the IR behavior would be completely different. It cannot be a conformal field theory.

Thus our results at the finite lattice size (up to $16^{3} \times 64$ ) are consistent with that the conformal window is $7 \leq N_{f} \leq 16$. However we do not exclude the possibility of the "walking scenario" that the RG beta function is anomalously small near the edge of the conformal window (e.g. $N_{f}=7$ or 8), and for a larger $N$ an undiscovered chiral phase transition point happens to appear at some value of $\beta$ and the chiral phase transition eventually occurs in the infinite $N$ limit.

We would like to stress that a conformal field theory is completely different from QCD in the point that there is no dimensional parameter such as $\Lambda_{\mathrm{QCD}}$. In QCD if $N a$ is large enough compared with $\Lambda_{\mathrm{QCD}}$, boundary effects can be neglected and it can be assumed the limit $N=\infty$ is taken. However the boundary effects are essential even at any large lattice $N$ in the conformal field theories because there is no other natural scale to compare. Note that our propagators are functions of the scaled time $\tau$ which takes value $0.0 \leq \tau \leq 1.0$. Clearly the function depends on the boundary condition as well as the aspect ratio even if we take $N \rightarrow \infty$ limit. Of course, to be clear, this does not mean that the local physics of the conformal field theory depends on the boundary conditions we use. We note the zero momentum propagator in our definition (1) may not be a local variable because we have summed over spatial coordinates before taking the continuum limit.

In the near future we would like to perform the program with larger lattice sizes and more statistics to derive the anomalous mass dimension using Eq5 and the relation of the eigenvalue density of the Dirac-Wilson operator and the anomalous index [8] [12][13]. It would be intriguing to compare them with the value from the unparticle meson model.

The calculations were performed with HA-PACS computer at CCS, University of Tsukuba and SR16000 at KEK. We would like to thank members of CCS and KEK for their strong support for this work.

\section{References}

[1] Y. Nakayama, arXiv:1302.0884 [hep-th].

[2] J. Kuti, PoS(LATTICE 2013)004,

E. Itou, PoS(LATTICE 2013)005,

J. Giedt, PoS(LATTICE2012)006,

L. Del Debbio, Int. J. Mod. Phys. A29 (2014) 25, 1445006; and references therein. 
[3] K.-I. Ishikawa, Y. Iwasaki, Y. Nakayama and T. Yoshie, Phys. Lett. B 748, 289 (2015) [arXiv:1503.02359 [hep-lat]].

[4] T. Banks and A. Zaks, Nucl. Phys. B196 (1982) 189.

[5] Y. Iwasaki, K. Kanaya, S. Kaya, S. Sakai and T. Yoshie, Phys. Rev. D 69, 014507 (2004)

[6] K.-I. Ishikawa, Y. Iwasaki, Yu Nakayama and T. Yoshie, Phys. Rev. D 89114503 (2014); arXiv:1304.4345; Phys. Rev. D 87071503 (2013) arXiv:1301.4785.

[7] Y. Iwasaki, UTHEP-118(1983); arXiv:1111.7059

[8] L. Del Debbio and R. Zwicky, Phys. Rev. D 82, 014502 (2010).

[9] M. Hayakawa, et al., PoS LATTICE 2010 (2010) 325.

[10] M. A. Clark and A. D. Kennedy, Phys. Rev. Lett. 98 (2007) 051601.

[11] Y. Iwasaki, K. Kanaya, S. Kaya, S. Sakai, and T. Yoshié, Phys. Rev. D54 (1996) 7010.

[12] A. Patella, Phys. Rev. D 86, 025006 (2012).

[13] A. Cheng, A. Hasenfratz, G. Petropoulos, D. Schaich, JHEP 1307 (2013) 061 